Brazilian Journal

of Chemical

ISSN 0104-6632

Engineering

Vol. 19, No. 03, pp. 299 - 306, July - September 2002

\title{
ANALYSIS AND OPTIMIZATION OF GAS- CENTRIFUGAL SEPARATION OF URANIUM ISOTOPES BY NEURAL NETWORKS
}

\author{
S.C.P.Migliavacca ${ }^{1}$, C.Rodrigues ${ }^{1}$ and C.A.O.Nascimento ${ }^{2 *}$ \\ ${ }^{1}$ IPEN - Instituto de Pesquisa Energética e Nucleares \\ ${ }^{2}$ Department of Chemical Engineering, Polytechnic School of University \\ of São Paulo, CEP 05508-900, São Paulo, S.P., Brazil \\ E-mail: oller@usp.br
}

(Received: September 5, 2001 ; Accepted: June 13, 2002)

\begin{abstract}
Neural networks are an attractive alternative for modeling complex problems with too many difficulties to be solved by a phenomenological model. A feed-forward neural network was used to model a gas-centrifugal separation of uranium isotopes. The prediction showed good agreement with the experimental data. An optimization study was carried out. The optimal operational condition was tested by a new experiment and a difference of less than $1 \%$ was found.

Keywords: neural networks, isotope separation, gas centrifugation, optimization, uranium isotopes, modeling.
\end{abstract}

\section{INTRODUCTION}

Prediction of the separation of uranium isotopes by gas centrifuge process employing mathematical models is quite difficult. Calculations require the simultaneous solution of the gas motion equations (continuity equation, the Navier-Stokes equations and energy equation) and the diffusion equation. The diffusion equation may be solved independently of the gas motion equations, since the difference in mass between uranium isotopes is far smaller than the average of the masses, after the gas motion equations are solved.

The separation analysis of the countercurrent centrifuge was first defined by Cohen (1951) in the 40's through solution of the diffusion equation using the method developed by Furry et al. (1939) for the thermal diffusion column. This became a classic solution, known as the Cohen-Onsager equation. This solution made many simplifications, such as a constant axial countercurrent flow and a radial averaged concentration. These simplifying hypotheses, which introduce errors into the results as compared with actual centrifuges, have gradually been improved (Olander, 1972). Recent works have resorted two-dimensional analytical or numerical analysis (Soubbaramayer, 1979; Makihara and Ito, 1988). Kai (1989) reviewed his studies conducted at the Power Reactor and Nuclear Fuel Development Corp. (PNC) with a two-dimensional numerical model, considering the nonlinear system, but also emphasizing the difficulties of predicting the separative performance of a real gas centrifuge.

Analytical or numerical solution of these modelbased equations, always requires the use of approximations, especially concerning the boundary conditions of the internal components. Consequently, none of the existing methods of calculation are valid for an actual centrifuge, although they are valuable for understanding the physical phenomena that take place within the gas centrifuge.

Using Zippe's data (1960), Migliavacca et al. (1999) simulated the separation of uranium isotopes

*To whom correspondence should be addressed 
with a neural network model. Good agreement was achieved.

In the present work the use of neural networks for the simulation and optimization of the separative performance of a gas centrifuge is proposed using new experimental data acquired from Centro Tecnológico da Marinha em São Paulo and IPEN.

\section{NEURAL NETWORK}

Neural networks are one of the fastest growing areas of artificial intelligence, particularly in chemical and nuclear engineering. Many applications in modeling and optimization of chemical processes are available in the open literature (Nascimento et al., 1999; Nascimento et al., 2000). In nuclear technology, the use of neural networks dates bake to the end of the 80's. They have been widely used in high energy physics (Denby, 1992) and in nuclear power plants (Eryürek et.al., 1991).

The application of neural networks in the simulation of chemical and nuclear processes, specifically in isotope separation by the gas centrifuge, is at considerable interest due to the nonlinearity of the process. This technique results in numerical models that are valid for actual centrifuges, thereby avoiding some of the difficulties encountered with the phenomenological model. The success of this kind of modeling depends largely on knowledge of the main variables affecting the process and the availability of a good database with the necessary information on the desired domain. The neural network used in this work was the multilayer feedforward network with the threelayered network, consisting of an input layer, a hidden layer and an output layer. The input layer consists of $n i+1$ neurons, where $n i$ is the number of input variables, and there is no processing in this layer. Besides the inputs, a bias is introduced into the network. The inputs are normalized between 0.1 and 0.9 in order to improve the convergence process. The number of neurons in the hidden layer is defined by the user. According to Pollard et al. (1992), the final precision is only slightly sensitive to the number of neurons in the hidden layer beyond a minimum value. The output layer consists of a number of neurons equivalent to the number of outputs of the process.

The backpropagation algorithm was used for training the three-layered feedforward neural networks used in this work (Rumelhart \& McClelland, 1986). This algorithm is a generalization of the steepest descent method.

\section{SIMULATION OF A GAS CENTRIFUGE VIA NEURAL NETWORK}

The separation parameters of a gas centrifuge can be experimentally determined in a separation experiment. The centrifuge used was a three-pole centrifuge with the internal fluid flow driven by a stationary scoop at one end of the rotor and a rotating baffle at the other end. An orthogonal design of experiments was proposed to study the influence of four construction variables, namely the product and the waste scoops, the axial feed location and the dimension of the rotating baffle. A separation experiment was run for each arrangement proposed in the design of experiments. The neural network technique was applied to the results of one of the experiments proposed. In the separation experiment, the pressure of the feed, product and waste lines and the mass flows of product and waste were measured. The centrifuge was operated under different conditions, defined by the flow rates and the output pressure, and approximately equal flows of product and waste were assumed, in order to study the symmetric case of separation, defined by $\beta=\gamma$. For each operational condition, samples were taken and analyzed to obtain the abundance ratio of each stream processed by the gas centrifuge. The results for 58 experiments were obtained; each of them consisted of the values of the product and waste flow rates, the pressures in the feed line and in the product and waste throughput, and the abundance ratios of the enriched and depleted material.

A neural network approach for the centrifuge was then applied, using the following input variables:

$\mathrm{Q}_{\mathrm{P}}$ : mass flow of the product;

$\mathrm{Q}_{\mathrm{W}}$ : mass flow of the waste;

$\mathrm{p}_{\mathrm{P}}$ : $\quad$ pressure in the product line;

and output variables:

$\mathrm{p}_{\mathrm{F}}: \quad$ pressure in the feed line;

$\mathrm{p}_{\mathrm{W}}$ : pressure in the waste line;

$\mathrm{R}_{\mathrm{P}}$ : abundance ratio of the product;

$\mathrm{R}_{\mathrm{W}}$ : abundance ratio of the waste. 
The abundance ratio of the feed gas is that of naturally occurring uranium.

\section{Learning and Testing with the Neural Network}

The experimental data were divided into two groups: a learning set, with 29 data subsets, used for the process of training the neural network, and a test set, with 27 data subset, used for checking the training, which were selected randomly. Each data subset results from the mean value of three experimental determinations. A three-layered feedforward neural network was used and trained with the backpropagation algorithm. Four different networks were tested: networks with 5, 6, 7 and 8 neurons in the hidden layer. The numbers of presentations used to train the neural network were between 50,000 and 100,000 . Table 1 shows the errors calculated in each case for the learning set and for the test set.

Allowing for a larger number of presentations, it was verified that 100,000 presentations were enough to determine the configuration of the network. The convergence in this case showed an asymptotic behavior for both, learning and test set. With these results, a neural network with seven neurons in the hidden layer was chosen.

\section{Comparison of Experimental Versus Calculated Data}

After training the neural network, the weights were chosen to minimize the error in the test set. The primary variables used were pressures $\mathrm{p}_{\mathrm{F}}$ and $\mathrm{p}_{\mathrm{W}}$. However, the most important variables in practice are the separation factor, $\alpha$, and the separative power, $\delta \mathrm{U}$, that represents the separation performance of the centrifuge. The separative power

$\mathrm{U}$ is defined as the work required to separate a certain flow of material into two flows of different concentrations.
$\alpha=\frac{\mathrm{R}_{\mathrm{P}}}{\mathrm{R}_{\mathrm{W}}}$

$\delta \mathrm{U}=\mathrm{Q}_{\mathrm{P}} \frac{\mathrm{R}_{\mathrm{P}}-1}{\mathrm{R}_{\mathrm{P}}+1} \ln \left(\mathrm{R}_{\mathrm{P}}\right)+$

$+\mathrm{Q}_{\mathrm{W}} \frac{\mathrm{R}_{\mathrm{W}}-1}{\mathrm{R}_{\mathrm{W}}+1} \ln \left(\mathrm{R}_{\mathrm{W}}\right)-$

$-\left(Q_{P}+Q_{W}\right) \frac{R_{F}-1}{R_{F}+1} \ln \left(R_{F}\right)$

Figure 1 shows a diagram of the model proposed to calculate the separation parameters of the gas centrifuge using the trained neural network.

Comparisons of the primary calculated variables, $\mathrm{p}_{\mathrm{F}}$ and $\mathrm{p}_{\mathrm{W}}$, with the experimental values are shown in Figures 2 and 3, respectively. The agreement between the experimental and calculated values for those variables is satisfactory. Normally the training set is always fitted (neural networks as used in this work are referred to as universal approximators (Hornik et al., 1989). The most important, however, is the prediction that the neural network made with the test set.

Parameters $\alpha$ and $\delta U$ are calculated according to the diagram shown in Figure 1. The values of the abundance ratios are given by the neural network and the values of $\alpha$ and $\delta U$ are calculated with equations (1) and (2). Figures 4 and 5 show the comparisons of the experimental and calculated values for these variables. The reason for calculating the values of $\alpha$ and $\delta U$ by equations (1) and (2), and not directly from the neural network, was because they do not represent primary variables. Normally the values of $\alpha$ and $\delta U$ are used for design purposes. The calculated values of these variables are in good agreement with the experimental values for both the learning and test sets. A good fitting in the test set means that the neural network can represent well the response surface of the real problem.

Table 1: Total errors calculated after the training of neural networks with different numbers of neurons in the hidden layer

\begin{tabular}{|c|c|c|c|c|}
\hline & \multicolumn{2}{|c|}{ LEARNING SET } & \multicolumn{2}{c|}{ TEST SET } \\
\hline NH & N. SETS & RMST & N. SETS & RMSTT \\
\hline 5 & 100000 & 0.123 & 11000 & 0.338 \\
\hline 6 & 100000 & 0.070 & 100000 & 0.185 \\
\hline 7 & 100000 & 0.056 & 100000 & 0.144 \\
\hline 8 & 50000 & 0.114 & 100000 & 0.313 \\
\hline
\end{tabular}




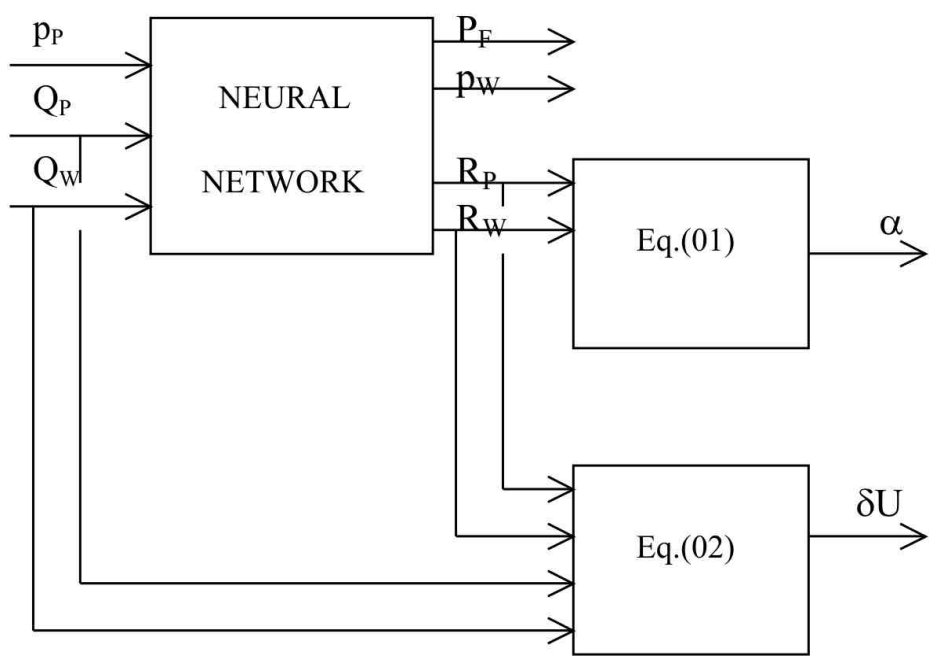

Figure 1: Model for the calculation of the parameters of the gas centrifuge using a neural network

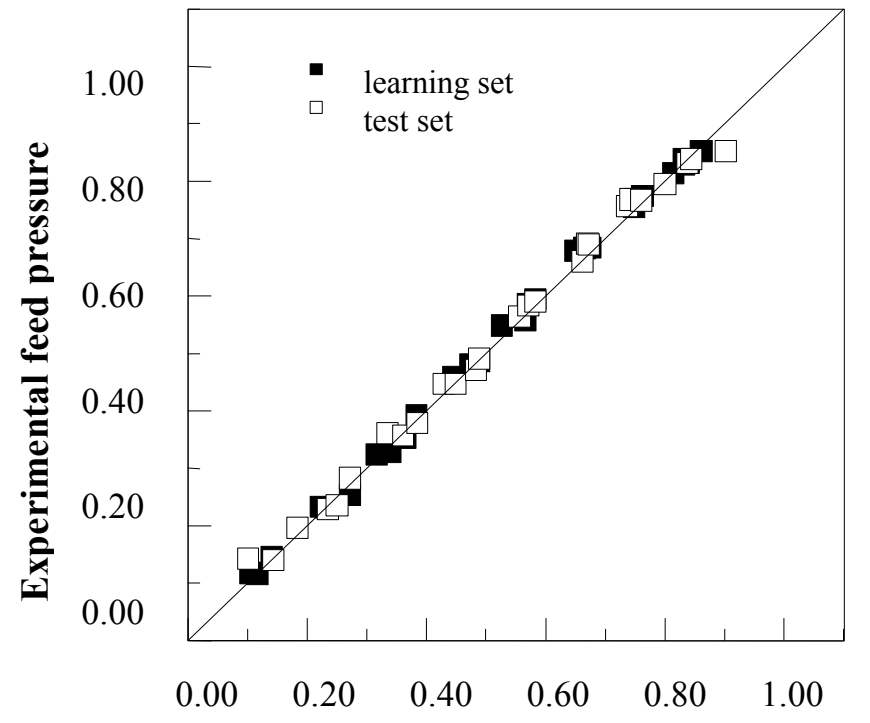

Calculated feed pressure

Figure 2: Comparison of the experimental and the neural network calculated values for the pressure in the feed line, $\mathrm{p}_{\mathrm{F}}$ (arbitrary units). 


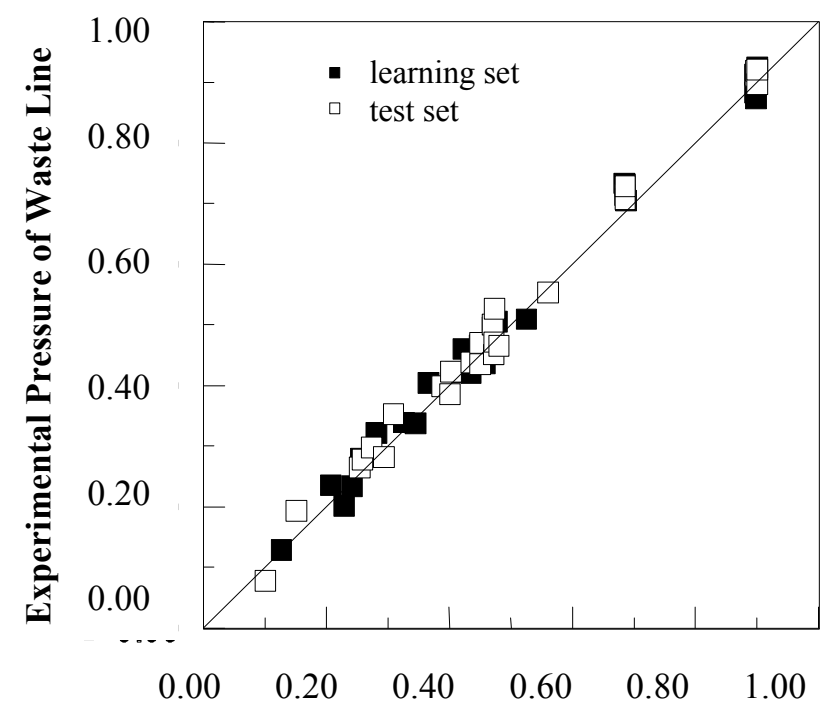

\section{Calculated Pressure of Waste Line}

Figure 3: Comparison of the experimental and the neural network calculated values for the pressure in the waste line, $\mathrm{p}_{\mathrm{W}}$ (arbitrary units).

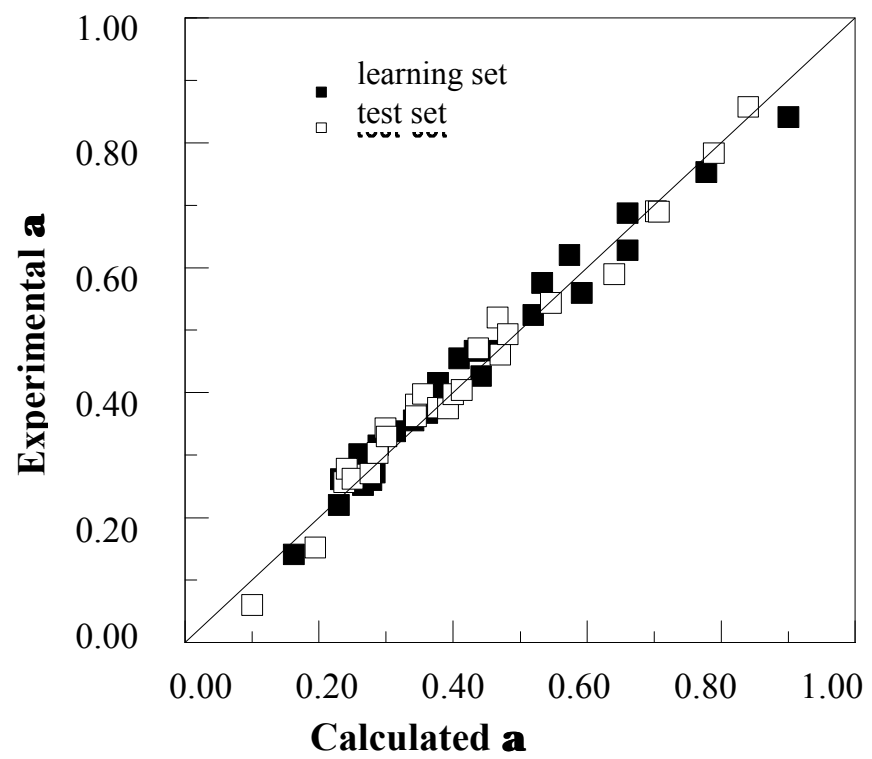

Figure 4: Comparison of the experimental and the neural network calculated values for the separation factor, $\alpha$ 


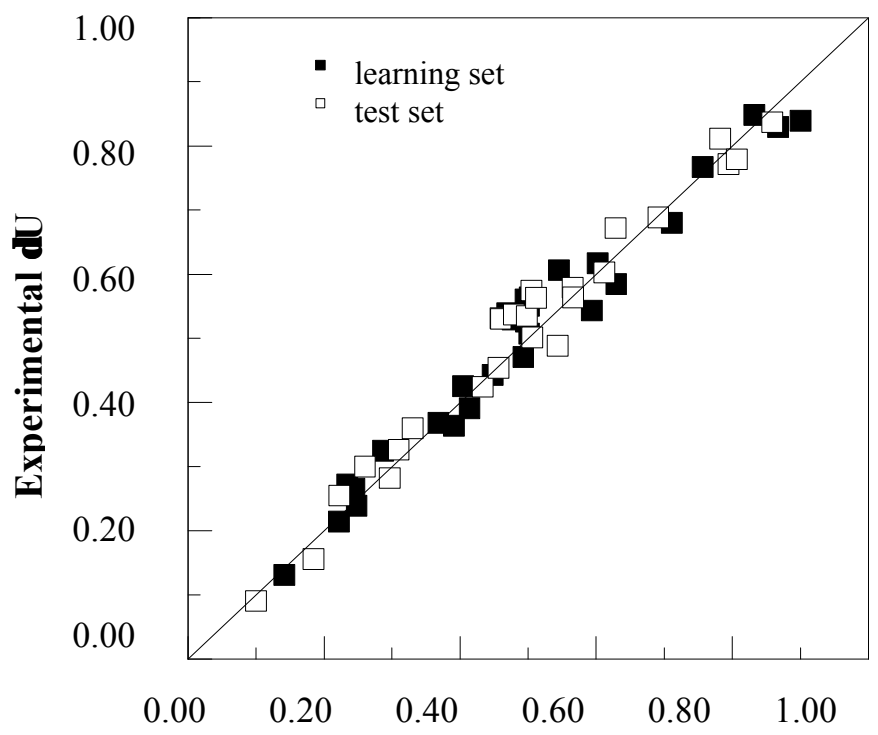

Calculated $\delta \mathbf{U}$

Figure 5: Comparison of the experimental and the neural network calculated values for the separation power, $\delta \mathrm{U}$

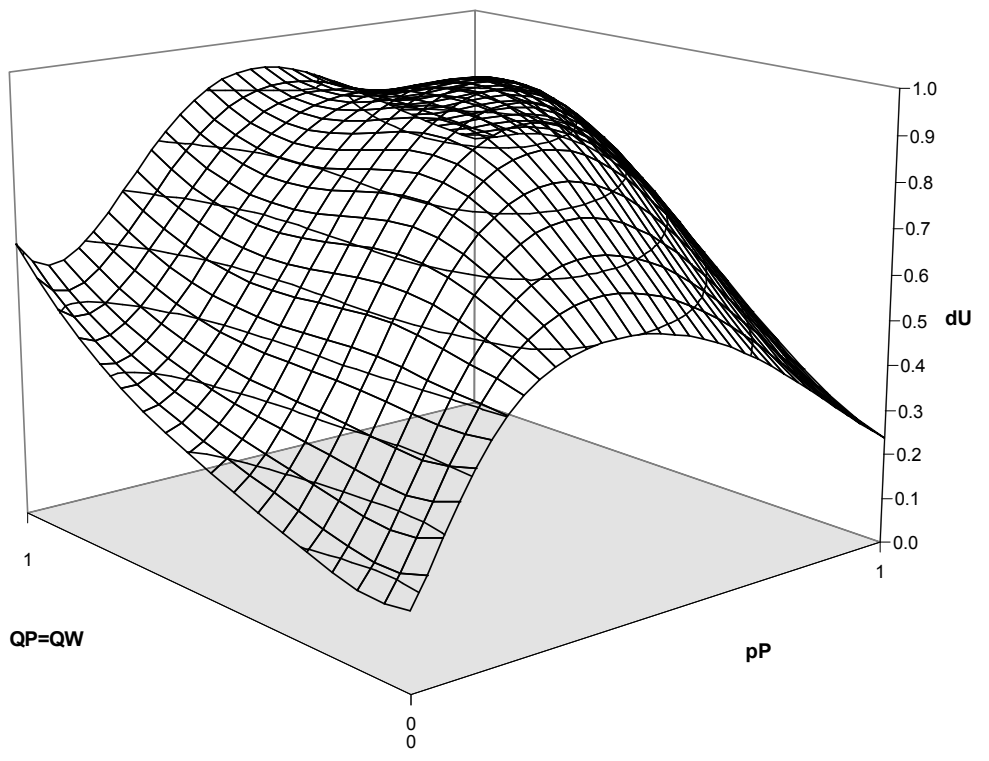

Figure 6: Response surface for the separative power of the centrifuge 


\section{OPTIMIZATION OF THE CENTRIFUGE}

Once the neural network was trained to represent the centrifuge, the solution of the problem was mapped on a very fine grid in the domain of the learning set data. Figure 6 shows a typical surface of the solution of the separative power as a function of flows $\mathrm{Q}_{\mathrm{P}}=\mathrm{Q}_{\mathrm{W}}$ and pressure $\mathrm{p}_{\mathrm{P}}$. With these results, the conditions under which the separative power is maximized can be found by inspection of the surface shown in Figure 6.

A new experiment was then carried out under the conditions of the optimized $\delta \mathrm{U}$, predicted by the simulated surface of $\delta U$ as a function of $Q_{P}$ and $p_{p}$. The difference between the experimental results and the values calculated using the neural network model was close to $1 \%$.

\section{CONCLUSION}

Modeling by neural networks has been shown to be an important tool to simulate and optimize the separation of uranium isotopes process. Experimental data of good quality is fundamental for obtaining reasonable results. The neural network model obtained is valid only for the centrifuge used in the separation experiment. In the present case, the experimental data covered a large domain of variables employed in the model. This fact is very important in order to be confident that the neural network has learned most of the nonlinear information in the process. Separation of the uranium isotopes was optimized by direct inspection of the surface response generated by the neural network. The optimal condition was checked by an independent new experiment and there was a difference less than $1 \%$ between the experimental value and the prediction.

\section{NOMECLATURE}

ni number of input variables in the neural network model

$\mathrm{NH}$ number of neurons in the hidden layer

N. SETS number of presentations of the data to the network

$\mathrm{p}_{\mathrm{F}} \quad$ pressure in the feed line

$\mathrm{p}_{\mathrm{P}} \quad$ pressure in the product line

$\mathrm{p}_{\mathrm{W}} \quad$ pressure in the waste line

$\begin{array}{ll}\mathrm{Q}_{\mathrm{P}} & \begin{array}{l}\text { mass flow of the product } \\ \text { mass flow of the waste } \\ \mathrm{Q}_{\mathrm{W}}\end{array} \\ \mathrm{r} & \begin{array}{l}\text { number of input/output pairs in the } \\ \text { learning set }\end{array} \\ \mathrm{RMST} & \begin{array}{l}\text { sum of square errors between } \\ \text { experimental and calculated values of } \\ \text { the learning set }\end{array} \\ \text { RMSTT } & \begin{array}{l}\text { sum of square errors between } \\ \text { experimental and calculated values of } \\ \text { the test set } \\ \text { abundance ratio of the product } \\ \text { abundance ratio of the waste }\end{array} \\ \mathrm{R}_{\mathrm{P}} & \end{array}$

\section{Greek Symbols}

$\begin{array}{ll}\alpha & \text { separation factor }\left(=\mathrm{R}_{\mathrm{P}} / \mathrm{R}_{\mathrm{W}}\right) \\ \beta & \text { head separation factor }\left(=\mathrm{R}_{\mathrm{P}} / \mathrm{R}_{\mathrm{F}}\right) \\ \gamma & \text { tail separation factor }\left(=\mathrm{R}_{\mathrm{F}} / \mathrm{R}_{\mathrm{W}}\right) \\ \delta \mathrm{U} & \text { separative power }\end{array}$

\section{ACKNOWLEDGMENT}

The authors would like to thank the Centro de Tecnologia da Marinha em São Paulo for allowing publication of this paper, FAPESP for sponsoring one of the authors and Prof. Frank Quina for revising the manuscript.

\section{REFERENCES}

Cohen, K., The Theory of Isotope Separation as Aapplied to the Large Scale Production of $\mathrm{U}^{235}$. McGraw-Hill, New York (1951).

Denby, B., Tutorial on Neural Network Applications in High Energy Physics: A 1992 Perspective. CONF-920172-7. 2nd. International Workshop on Software Engineering, Artificial Intelligence (AI) and Neural Nets for High Energy and Nuclear Physics. L’Agelonde, France (1992).

Eryürek, E. and Türckan, E., Neural Networks for Sensor Validation and Plant-wide Monitoring. ECN-RX-91-089, Netherlands Energy Research Foundation (ECN), (1991).

Furry, W.H., Jones, R.C. and Onsager, L., On the Theory of Isotope Separation by Thermal Diffusion. Phys. Rev., No. 55, 1083-1095 (1939). Hornik, K., Stinchcombe. M. and White, H., 
Multilayer Feedforward Networks are Universal Approximators. Neural Networks, vol. 2, 359-366 (1989).

Kai, T., Theoretical Research on Gas-Centrifugal Separation for Uranium Enrichment. J. Nucl. Sci. and Technol., vol. 26, No. 1, 157-160 (1989).

Makihara, H. and Ito, T., Separation Characteristics of Gas Centrifuges, Approximate Analyses of Separation Performance. J. Nucl. Scie. and Technol. vol. 25, No. 8, 649-666 (1988).

Migliavacca, S.C.P., Nascimento, C.A.O. and Rodrigues, C., Use of Neural Network for the Simulation of a Gas Centrifuge. J. Nucl. Sci. and Technol., vol. 36, No. 4, 364-370 (1999).

Nascimento, C.A.O., Giudici, R.and Scherbakoff, N., Modeling of Industrial Nylon-6,6 Polymerization Process in a Twin-Screw Extruder Reactor. II. Neural Networks and Hybrid Models. J. Applied Polymer Science, No. 72, 905-912 (1999).
Nascimento, C.A.O., Giudici, R. and Guardani, R., Neural Network Based Approach for Optimization of Industrial Chemical Processes. Comput. \& Chem. Engng., No. 24, 2303-2314 (2000).

Olander, D.R., Technical Basis of the Gas Centrifuge. Adv. Nucl. Sci. Technol., No. 6, 105174 (1972).

Pollard, J.F., Broussard, M.R., Garrison, D.B. and San,K.Y., Process Identification Using Neural Networks. Computers \& Chemical Engineering, vol. 16, No. 4, 253-270 (1992).

Rumelhart, D. and McClelland, J., Parallel Distributed Processing Explorations in the Microstructure of Cognition, vol. 1, cap. 8, MIT, Cambridge, Mass. (1986).

Soubbaramayer, Centrifugation. In: VILLANI, S., ed. Uranium Enrichment. Springer Verlag, Berlin, p. 183- 243 (1979).

Zippe, G., The Development of Short Bowl Ultracentrifuges. Final report ORO-315 (1960). 